\title{
Hidatidosis hepática. Cirugía radical vs. no radical: 22 años de experiencia
}

\author{
P. Priego, J. Nuño, P. López Hervás, A. López Buenadicha, R. Peromingo, J. Die, G. Rodríguez y V. Fresneda \\ Departamento de Cirugía General y Digestivo. Hospital Ramón y Cajal. Madrid
}

\section{RESUMEN}

Introducción: la incidencia de la hidatidosis hepática ha disminuido notablemente en los últimos años gracias a las medidas de prevención adoptadas para interrumpir la transmisión del parásito. Con todo, la cirugía continúa siendo el tratamiento de elección, si bien su modalidad es todavía motivo de controversia.

Objetivos: el objetivo de este trabajo es evaluar los resultados obtenidos en el tratamiento de esta patología a lo largo de más de dos décadas, atendiendo a la modalidad quirúrgica empleada ya fuese cirugía radical o no radical.

Material y métodos: se analizaron un total de 372 pacientes intervenidos por quiste hepático hidatídico (QHH) entre 19832005 en el Hospital Ramón y Cajal. En162 se efectúa una cirugía radical $(43,5 \%)$ y en 210 una no radical $(56,5 \%)$.

Resultados: tanto la estancia media hospitalaria $(8,65$ días $v s$. 14,9 días) como la morbilidad $(13,3$ vs. $31,4 \%, p<0,001)$ y la mortalidad (0 vs. 3,8\%, p < 0,01) fueron menores en el grupo de cirugía radical. La tasa de recidiva fue del $1,85 \%$ tras un abordaje radical frente al 11,9\% en los abordajes no radicales ( $p<0,0001)$.

Conclusión: la cirugía radical se asocia con una menor morbimortalidad, menor estancia hospitalaria y menor recidiva, constituyendo la técnica de elección en la hidatidosis hepática. Sin embargo, su aplicación debe atenerse a las características del paciente, la anatomía del quiste y el grado de experiencia del equipo quirúrgico.

Palabras clave: Quiste hidatídico. Tratamiento. Cirugía radical. Cirugía no radical.

\begin{abstract}
Objectives: the incidence of hepatic hydatidosis has remarkably decreased in the last years due to the preventive measures adopted to stop the transmission of the parasite. However, surgery carries on being the treatment of choice, although the surgical procedure is still a matter of controversy. The aim of the study was to evaluate the results obtained with the treatment of this condition after two decades according to surgical procedure type.

Material and methods: from 1983 to 2005, 372 patients were operated on for hepatic hydatidic cyst in Hospital Ramón y Cajal. Radical surgery was performed for 162 (43.5\%) and conservative surgery for 210 (56.5\%).

Results: average postoperative hospital stay (8.65 vs. 14.9 days), morbidity (13.3 vs. 31.4\%, p < 0.001), and mortality (0 vs. $3.8 \%, p<0.01$ ) were lower in the radical surgery group. Recurrence rate was $1.85 \%$ after radical surgery versus $11.9 \%$ in the conservative surgery group ( $p<0.0001)$.

Conclusion: radical surgery is associated with lower morbidity, mortality, postoperative hospital stay, and recurrence rates, and represents the treatment of choice for hepatic hydatidosis. However, its indication must depend on the patient characteristics, cyst anatomy, and surgical team experience.
\end{abstract}

Key words: Hydatidic cyst. Treatment. Radical surgery. Conservative surgery.

Priego P, Nuño J, López Hervás P, López Buenadicha A, Peromingo R, Die J, Rodríguez G, Fresneda V. Hidatidosis hepática. Cirugía radical us. no radical: 22 años de experiencia. Rev Esp Enferm Dig 2008; 100: 82-85.

Recibido: 27-08-07.

Aceptado: 15-11-07

Correspondencia: Pablo Priego Jiménez. C/ Fermín Caballero, 26, $1^{\circ}$ A. 16004 Cuenca.e-mail: papriego@hotmail.com

\section{INTRODUCCIÓN}

La hidatidosis hepática es una enfermedad producida por el crecimiento de la larva del Echinococcus granulosus en el cuerpo humano, que es un huésped intermedio en el desarrollo del parásito (1). 
Aunque tradicionalmente se ha considerado como una patología "benigna", su curso clínico variable, evolución incierta y posibilidad de complicaciones diversas hacen de esta patología una enfermedad potencialmente letal y con un porcentaje nada despreciable de mortalidad.

A pesar del empleo de agentes quimioterápicos como el mebendazol o el albendazol, la cirugía continúa siendo el tratamiento de elección de este tipo de patología (2,3).

Clásicamente los procedimientos quirúrgicos se han dividido en dos grupos: radicales y no radicales, siendo su elección controvertida. Los resultados obtenidos con las técnicas radicales han sido mejores en cuanto a tasa de recidiva, la estancia hospitalaria y la morbimortalidad, pero ello conlleva unas actuaciones más arriesgadas que pueden resultar comprometedoras para un paciente afecto de una patología considerada como benigna $(4,5)$.

El objetivo de este estudio es analizar nuestra experiencia en el tratamiento quirúrgico de la hidatidosis hepática atendiendo especialmente a la modalidad quirúrgica empleada.

\section{MATERIAL Y MÉTODOS}

Se ha efectuado un análisis retrospectivo de un total de 372 pacientes diagnosticados de hidatidosis hepática (HH) intervenidos en el Hospital Ramón y Cajal entre enero de 1983 y diciembre de 2005.

El diagnóstico se fundamentó en las pruebas de imágen (TAC, ecografía, RMN) y de laboratorio (eosinofilia, test función hepática), así como en los test serológicos (anticuerpos e Ig E frente al echinococco).

De acuerdo a la modalidad quirúrgica empleada, hemos considerado procedimientos radicales (CR): la quistoperiquistectomía total, quistoperiquistectomía subtotal (dejando sólo remanente muy pequeño de periquística), la quistorresección y la hepatectomía. Hemos considerado procedimientos no radicales (CNR) todos los restantes procedimientos en los cuales la periquística no ha sido extirpada.

La elección del procedimiento quirúrgico empleado fue adoptada por cada uno de los cirujanos del servicio y se atuvo fundamentalmente a los siguientes criterios: localización y tamaño del quiste, relaciones vasculares, número de quistes, comorbilidad del paciente y experiencia quirúrgica. En este sentido hemos diferenciado dos grupos: grupo A (especial dedicación a la cirugía hepática) y grupo B (sin especial dedicación a la misma).

Para el análisis estadístico de los resultados se utilizó el programa SPSS, analizando las variables cualitativas mediante el test de la Chi cuadrado y el de la probabilidad de Fisher. Se considera que el resultado es estadísticamente significativo cuando la $\mathrm{p}<0,05$.

\section{RESULTADOS}

De los 372 pacientes, 45 habían sido intervenidos previamente por $\mathrm{HH}$, habiéndose realizado dicha cirugía entre 1 y 42 años antes (media 13,8 años). En 7 pacientes el diagnóstico de quiste hidatídico se asoció al de hipertensión portal, tanto por cirrosis biliar como por cavernoma. Durante el acto quirúrgico, se constataron 8 casos de reacción urticariforme y 4 de shock anafiláctico; hubo 2 embolismos pulmonares hidatídicos y un paciente desarrolló paraplejia durante su seguimiento en relación con hidatidosis vertebral.

Se efectuó CR en 162 pacientes $(43,5 \%)$ y CNR en $210(56,5 \%)$. La modalidad quirúrgica empleada por cada grupo de cirujanos aparece reflejada en la tabla I. Las figuras 1 y 2 muestran la evolución de aquella a lo largo del tiempo.

La distribución de los quistes hidatídicos en el hígado aparece reflejada en la figura 3 .

La estancia media hospitalaria fue menor en el grupo en el cual se efectuó CR (8,6 vs. 14,9 días), con rangos de 3-24 días y 6-29 días respectivamente.

La tasa global de complicaciones fue menor en el grupo de CR $(13,3 \%)$ frente al 31,4\% del grupo en el cual se efectuó una CNR ( $<<0,001$; OR: 3,07; IC95\% entre 1,7-5,3).

La tasa de mortalidad fue del $2,15 \%$, siendo menor en el grupo de CR $(0 \%)$ frente al 3,8\% en el grupo de CNR ( $\mathrm{p}<0,01$; OR: 1,04; intervalo de confianza del 95\% entre $1,012-1,068)$. No se produjo ningún caso de mortalidad intraoperatoria en el grupo de CR, frente a dos en el gru-

Tabla I. Modalidad quirúrgica/grupo quirúrgico

\begin{tabular}{lcc}
\hline & Cirugía radical* $^{*}$ & Cirugía no radical \\
\hline Grupo A & $108(66,6 \%)$ & $69(32,8 \%)$ \\
Grupo B & $54(33,4 \%)$ & $141(67,2 \%)$ \\
\hline${ }^{*} \mathrm{p}<0,001$. &
\end{tabular}

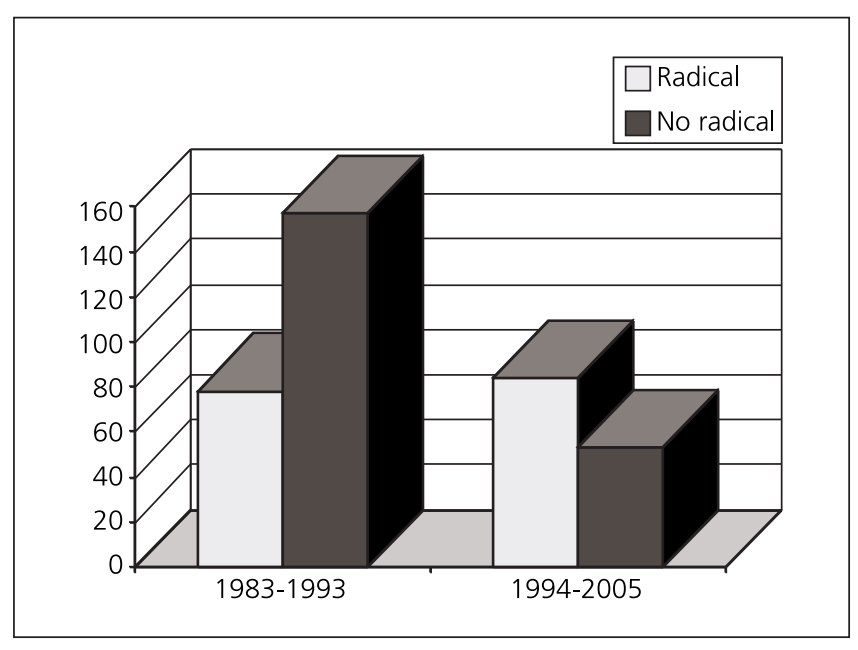

Fig. 1. Evolución de la modalidad quirúrgica I. 


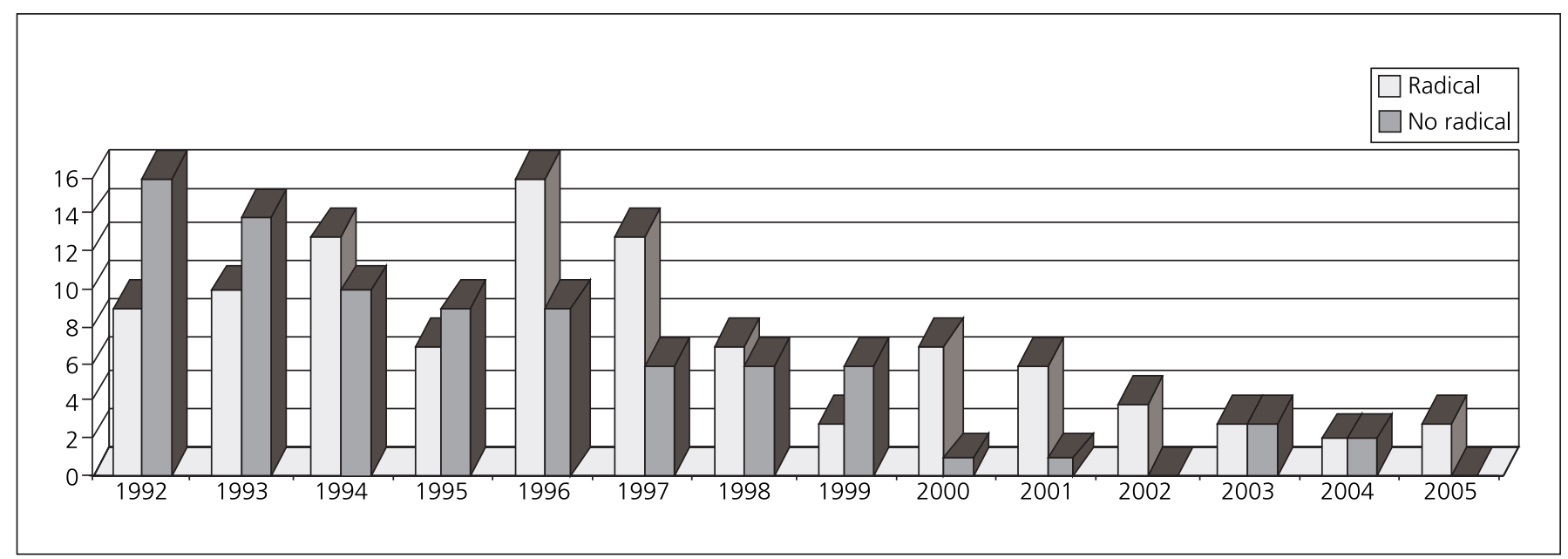

Fig. 2. Evolución de la modalidad quirúrgica II.

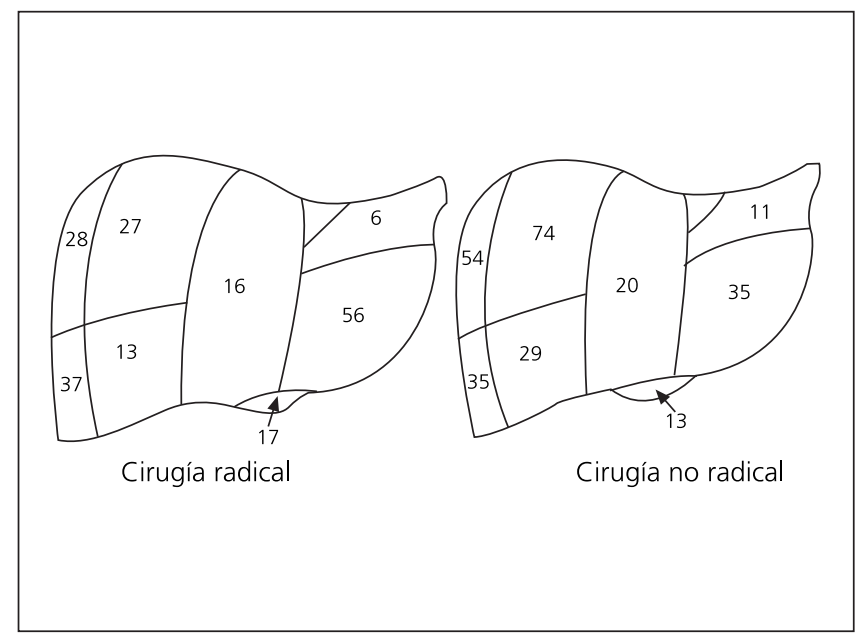

Fig. 3. Localización del quiste hidatídico.

Tabla II. Causas de mortalidad tardía

-Insuficiencia hepatorrenal

-Embolismo pulmonar

-Empiema pleural y sepsis

-Edema agudo pulmón

-TEP durante colangiografía

-Sepsis e insuficiencia hepática

po de CNR, que obedecieron a una hemorragia de la vena cava y a un embolismo pulmonar

Los restantes seis pacientes fallecieron tiempo después del alta hospitalaria por complicaciones de la enfermedad $(\mathrm{QHH})$, sus causas aparecen reflejadas en la tabla II.

En la tabla III se muestra el comparativo referente a la incidencia de fístula biliar, absceso abdominal, reingreso hospitalario y recidiva.

\section{DISCUSION}

Aunque tradicionalmente se ha considerado la hidatidosis hepática como una patología "benigna”, su curso clínico no siempre previsible, así como la posibilidad de complicaciones graves diversas, hacen de esta patología una enfermedad potencialmente letal.

La cirugía continúa siendo el tratamiento de elección, con una tasa de curación próxima al 90\% (2,3). La mejora en las condiciones socioeconómicas e higiénicas en muchos países ha conseguido reducir la incidencia de esta enfermedad endémica a nivel mundial. En nuestra casuística a lo largo de dos décadas, el número de intervenciones por $\mathrm{HH}$ ha sufrido un descenso próximo al $90 \%$. El manejo quirúrgico de estos pacientes ha sido motivo de controversia. No existe un grado de evidencia suficiente que permita afirmar la superioridad de una opción quirúrgica radical sobre la contraria (6).

Los defensores de un abordaje radical aducen que este conlleva menores tasas de recidiva, estancia hospitalaria y tasas de morbimortalidad (4,5,7-16). Sin embargo sus detractores defienden el abordaje no radical, al considerar que la $\mathrm{CR}$ conlleva maniobras quirúrgicas arriesgadas, que pueden comprometer la vida del paciente en una enfermedad considerada como benigna.

La tendencia actual es realizar un mayor número de CR. Esta ha sido también nuestra experiencia; así, en la primera década de nuestro estudio el $66 \%$ de los procedimientos fueron CNR (157 de 234 pacientes), mientras que esta tasa fue del $39 \%$ en la década posterior (53 de 138 pacientes).

Esta transformación obedece tanto a la especialización en la cirugía hepática como a los mejores resultados obtenidos con este tipo de procedimiento (CR), como lo reflejan los datos comparativos referentes a la estancia hospitalaria, morbilidad, mortalidad y recidiva. Esta experiencia es compartida por otros autores $(4,5,8-13,15)$. 
Tabla III. Morbilidad/Recidiva

\begin{tabular}{lcccc}
\hline \multicolumn{4}{c}{ Morbilidad/Recidiva } \\
\cline { 2 - 5 } & $\begin{array}{c}\text { Cirugía } \\
\text { radical }\end{array}$ & $\begin{array}{c}\text { Cirugía } \\
\text { no radical }\end{array}$ & Valor $p$ & OR (IC95\%) \\
\hline Fístula biliar & $4,3 \%$ & $25,6 \%$ & $p<0,0001$ & 7,66 \\
Absceso abdominal & $3 \%$ & $11,95 \%$ & $p<0,002$ & 4,24 \\
Reingreso & $3 \%$ & $12,58 \%$ & $p<0,001$ & 4,44 \\
Recidiva & $1,85 \%$ & $11,9 \%$ & $p<0,0001$ & 7,16 \\
\hline
\end{tabular}

Aun cuando la CR conlleva mejores resultados, consideramos que la misma no debe aplicarse de manera sistemática, sino que su indicación ha de basarse en las características de la $\mathrm{HH}$, la situación del paciente y la experiencia del cirujano. Así puede deducirse de nuestra propia experiencia donde, si bien la mayoría de los procedimientos de CR fueron llevados a cabo por el grupo de cirujanos con mayor experiencia en cirugía hepática, un tercio de las intervenciones efectuadas por este grupo fueron técnicas de CNR.

$\mathrm{El}$ auge experimentado por la cirugía laparoscópica en los últimos años se ha trasladado también al tratamiento del quiste hidatídico con buenos resultados (17-23). Sin embargo todavía no son precisas ni sus indicaciones ni sus resultados a largo plazo.

Como conclusión, recalcar que la cirugía radical se asocia con una menor tasa de morbimortalidad, estancia hospitalaria y recidiva, constituyendo la técnica de elección en la hidatidosis hepática. Sin embargo no se debe aplicar sistemáticamente a todos los pacientes, debiendo tener en cuenta las características del paciente, la anatomía del quiste y grado de experiencia del cirujano.

\section{BIBLIOGRAFÍA}

1. Moreno González E. Quistes hepáticos. En: Cantero B, editor. Tratado de Cirugía. Vol. 2. Barcelona: Editorial Toray; 1989. p. 237899.

2. Menezes da Silva A. Hydatid cyst of the liver-criteria for the selection of appropriate treatment. Acta Trop 2003; 85: 237.

3. WHO; Informal Working Group on Echinococcosis. Guidelines for the treatment of cystic and alveolar echinococcosis in humans. Bull
World Health Organ 1996; 74: 231

4. Di Matteo G, Bove A, Chiarini S, Capuano LG, De Antoni E, Lanzi $\mathrm{G}$, et al. Hepatic echinococcus disease: Our experience over 22 years. Hepatogastroenterology 1996; 43: 1562-5.

5. Alonso Casado O, Moreno González E, Loinaz Segurola C, Gimeno Calvo A, González Pinto I, Pérez Saborido B, et al. Results of 22 years of experience in radical surgical treatment of hepatic hydatid cysts. Hepatogastroenterology 2001; 48: 235-43.

6. Dziri C, Haouet K, Fingerhut A. Treatment of hydatid cyst of the liver: Where is the evidence? World J Surg 2004; 28 (8): 731-6.

7. Alfieri S, Doglietto GB, Pacelli F, Costamagna G, Carriero C, Mutignami M, et al. Radical surgery for liver hydatid disease: A study of 89 consecutive patients. Hepatogastroenterology 1997; 44: 496-500.

8. Puliga A, Sulius R, Pala M, Sechi R, Pietrangeli M. Surgical treatment of hydatid liver cysts: 20 more years of experience. Chir Ital 2003; 55 (4): 533-40.

9. Mosca F, Stracqualursi F, Persi A, Angilello A, Latteri S. Surgical treatment of hepatic hydatidosis. Analysis of results of conservative and radical surgery in our experience. Chir Ital 2003; 55 (1): 1-12.

10. Tasev V, Dimitrova V, Draganov K, Bulanov D, Popadiin N, Gaidarski R. Hepatic echinococcosis: Radical or conservative surgical treatment. Khirurgiia (Sofiia) 2002; 58 (2): 10-3.

11. Moreno González E, Jover Navalon JM, Landa García JL, et al. Surgical management of liver hydatidosis: 10 years of experience with 269 patients. Ital J Surg Sci 1985; 15: 267-73.

12. Berrada S, Essadki B, Zerouali NO. Hydatid cyst of the liver: Treatment by resection of the cyst wall: Our experience of a series of 495 cases. Ann Chir 1993; 47: 510-2.

13. Magistrelli P, Masetti R, Coppola R, et al. Surgical treatment of hydatid disease of the liver. A 20-year experience. Arch Surg 1991; 126: 518.

14. Behrns KE, Van Heerden JA. Surgical management of hepatic hydatid disease. Mayo Clin Proc 1991; 66: 1193.

15. Balik AA, Basoglu M, Celebi F, et al. Surgical treatment of hydatid disease of the liver: Review of 304 cases. Arch Surg 1999; 134: 166.

16. Gollackner B, Langle F, Auer H, Maier A, Mittlbock M, Agstner I, et al. Radical surgical therapy of abdominal cystic hydatid disease: Factors of recurrence. World J Surg 2000; 24 (6): 717-21.

17. Emel'ianov SI, Khamidov MA. Laparoscopic treatment of hydatid liver cysts. Khirurgia Mosk 2000; 11: 32-4.

18. Khoury G, Abiad F, Geagea T, et al. Laparoscopic treatment of hydatid cysts of the liver and spleen. Surg Endosc 2000; 14: 243-5.

19. Manterola C, Fernández O, Muñoz S, et al. Laparoscopic pericystectomy for liver hydatid cysts. Surg Endosc 2002; 16: 521-4.

20. Bickel A, Loberant N, Singer-Jordan J, et al. The laparoscopic approach to abdominal hydatid cysts: A prospective nonselective study using the isolated hypobaric technique. Arch Surg 2001; 136: 789-95.

21. Kayaalp C. Evacuation of hydatid liver cysts using laparoscopic trocar. World J Surg 2002; 26: 1324-7.

22. Ertem M, Karahasanoglu T, Yavuz N, Erguney S. Laparoscopically treated liver hydatid cysts. Arch Surg 2002; 137: 1170-3.

23. Papanivelu C, Jani K, Malladi V, Senthilkumar R, Rajan PS, Sendhilkumar K, et al. Laparoscopic management of hepatic hydatid disease. JSLS 2006; 10 (1): 56-62. 